empfiehlt Landolt ${ }^{1}$ ) einen Zusatz von Chlorkalium, zum Beispiel in Gestalt der Rückstände der früheren Sauerstoffbereitung. Die constante Entwicklung bedarf in diesem Fall keiner weiteren Aufsicht.

Zur Prüfung der Salzsäure auf freies Chlor empfiehlt Kupffer$\mathrm{schläge}{ }^{2}$ ) die Säure mit metallischem Kupfer in Berührung zu bringen, nach einiger Zeit fast zu neutralisiren und mit Ammoniak, besser mit Ferrocyankalium, auf gelöstes Kupfer zu prüfen. Chlorfreie Salzsäure löst Kapfer erst bei $200^{\circ} \mathrm{C}$.

Durch Behandlung der Salzsäure mit Phosphor und nachfolgende Prüfung mit molybdänsaurem Ammon lässt sich Chlor ebenfalls nachweisen, doch ist die erste Methode viel zuverlässiger und exacter als die letztere.

Ueber die Aufbewahrung von Schwefelwasserstoffwasser hat A. Schneider ${ }^{3}$ ) Versuche angestellt, welche die bekannte Thatsache bestätigen, dass Abschluss von Licht und hermetischer Verschluss der Flaschen wesentliche Erfordernisse sind, um das Reagens längere Zeit wirksam zu erhalten. Der Verfasser empfiehlt am meisten schwarze Glasflaschen mit Glasstöpsel, welche mit Vaselin nicht nur eingefettet, sondern dick eingeschmiert sind. Den Einfluss der Luft bei Abschluss des Lichtes hält Schneider für wenig schädlich, wenn nur die Verdunstung des Schwefelwasserstoffs vermieden wird.

\title{
II. Chemische Analyse anorganischer Körper.
} Von

\section{E. Hintz, unter Mitwirkung von H. Weber.}

Zur Elektrolyse. Nach Thomas Moore ${ }^{4}$ ) lässt sich eine Reihe von Metallen auf elektrolytischem Wege bestimmen, indem man dieselben bei Gegenwart von Phosphorsäure abscheidet; die Phosphorsäurelösung bereitet der Verfasser durch Auflösen von glasiger Phosphorsäure ${ }^{5}$ ).

1) Berg- u. Hüttenm. Zeitung 49, 428.

2) Bull. de la soc. chim. de Paris [3. Serie] 2, 134.

3) Pharm. Centralballe [N. F.] 9, 118.

4) Chem. News 53, 209.

5) Acidum phosphoricum glaciale. 